\title{
Information collection system of duck products based on loT
}

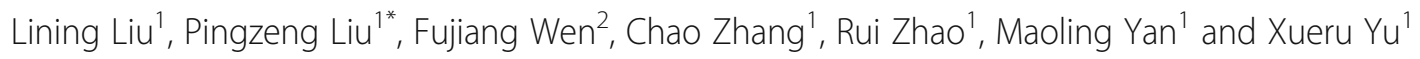

\begin{abstract}
In view of the problems existing in the processing of duck products, such as complicated technology, difficulties in information collection and information linkage, and lack of dedicated information collection equipment, the duck product traceability information of the Institute of Things automatic collection system was developed. Acquisition system is mainly composed of sensing terminals, bus structure, and host computer management system. Perceiving the terminal can automatically perceive the information of each link and transmit the perception information to the upper computer management system through the bus. The upper computer management system realizes the functions of storing the sensing information, intelligent analysis, and alarm prompting. The long-term operation results show that the system performance is stable and reliable; the collection of data is efficient, complete, and accurate; and the degree of automation of the system is high, which significantly improves the product quality and safety supervision capabilities of the company.
\end{abstract}

Keywords: Information collection system, Duck product traceability, loT, Industrial chain, RFID

\section{Introduction}

With the gradual improvement of people's living standards, food safety has attracted more and more attention. To achieve the purpose of effective management of agricultural product quality and safety, several relevant laws have been published on traceability of livestock and poultry product quality and safety in many countries. It is an inevitable choice for food production and processing companies to improve product quality and safety management capabilities and ensure food quality and safety through the application of food quality safety traceability systems. To achieve product quality and safety traceability, automatic collection of product quality and safety-related information is the key. At present, the automatic collection of product quality information by duck processing enterprises faces many difficulties. For example, the industry chain is long, the processing flow is complex, and it is difficult to automatically collect quality and safety information. Therefore, to achieve the quality and safety traceback of duck products, automatic collection of duck bird product quality and safety information is the key. With the development of the Internet of Things technology, the

\footnotetext{
* Correspondence: Ipz8565@126.com

${ }^{1}$ College of Information Science and Engineering, Shandong Agricultural University, Tai'an 271018, China

Full list of author information is available at the end of the article
}

widespread application of radio frequency identification technology and QR code identification technology provides support for the automatic collection of information related to quality and safety of duck products [1-3].

For the problem of obtaining quality information of livestock and poultry products, domestic and foreign scholars have conducted a large number of studies [4-9]. Mc Inerney et al. used the GS1 bar code technology to mark individual eggs and achieved accurate egg individual information [10]. Stanford et al. used electronic identification technology and DNA fingerprinting technology for individual labeling to achieve accurate collection of individual livestock and poultry information [11]. Sugiura et al. achieved the marking of livestock information through special ear tags, which enabled the rapid acquisition of livestock information, which laid the foundation for realizing individual animal traceability [12]. Feng et al. used RFID tags as a mark for cattle and sheep products and achieved the acquisition of cattle and sheep breeding information through PAD devices with RFID read-write capabilities [13].

Through domestic and foreign literature analysis, it can be seen that the traceability information first uses the QR code, RFID tags, and DNA as product identifiers and then obtains product information through manual or semi-automatic methods. The improvement of information acquisition capabilities has significantly 
improved the quality and safety management capabilities of products, but at the same time, there are still many problems in information collection, such as the low degree of automation of information acquisition, the lack of seamless linking of information, and the acquisition of information with "large granularity" [14-16].

\section{Methods}

\subsection{Research object}

In this study, a duck production scale processing plant was used as a research model, and duck individual and terminal products were taken as the research objects to study the data collection scheme of the duck product processing link.

\subsection{Information acquisition equipment}

This study combines the actual processing environment and processing methods in the processing field, adopts an RFID reader as the main method, and combines a variety of information acquisition methods such as a weight sensor, a number sensor, and a sweeping code gun to achieve seamless coverage of the entire processing link.

\subsection{Traceability information}

In the process of processing, the information to be obtained includes information on the source of raw materials, information on the responsible persons of each step, processing step information, product batch information, time information, and quality inspection information.

\section{Industrial chain processing flow analysis}

Through the analysis of the company's production processes, the duck industry is mainly composed of five links: aquaculture, processing, warehousing, logistics, and sales. The poultry industry structure and information flow chart are shown in Fig. 1. As shown in Fig. 1, the duck product quality and safety information starts

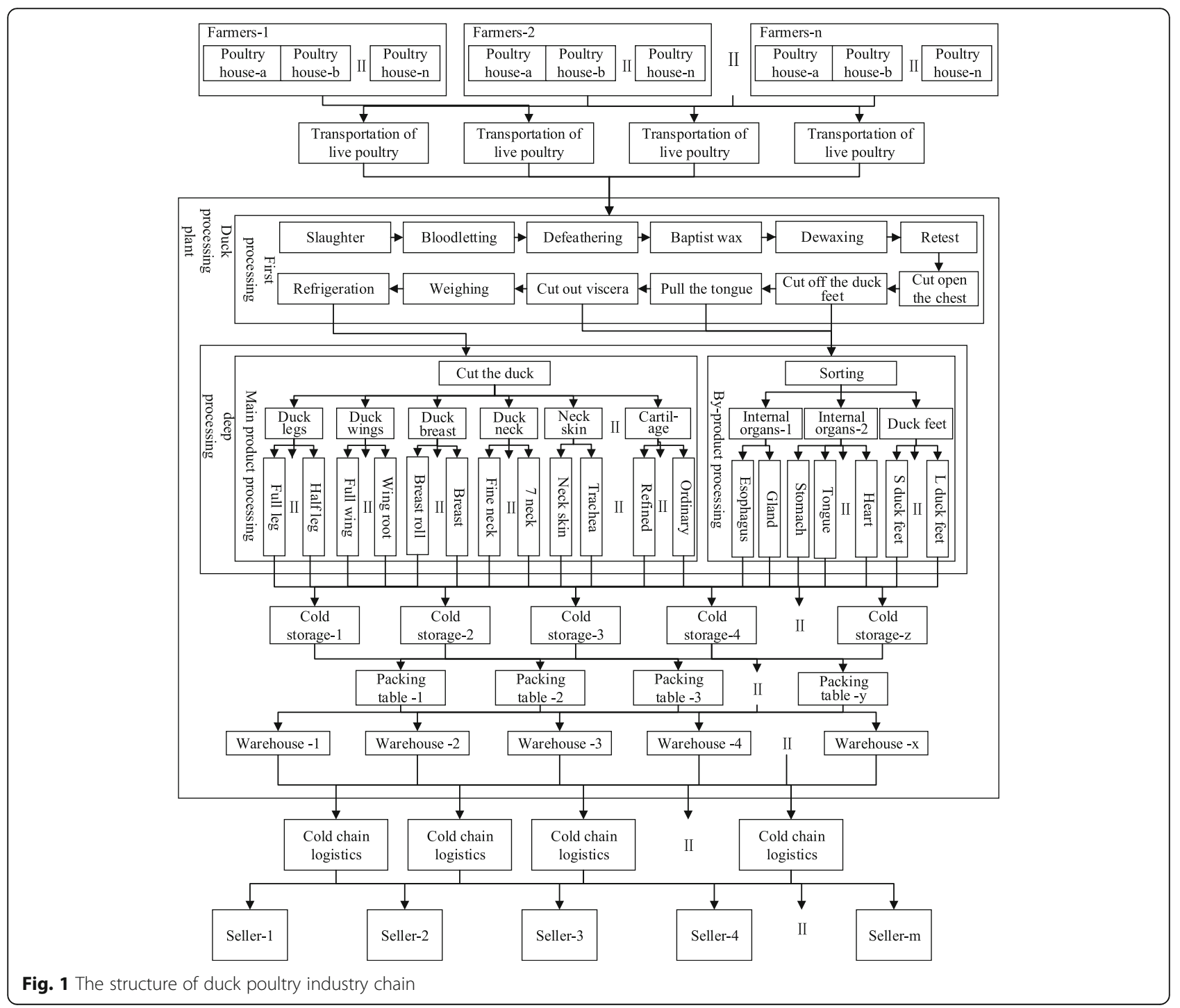


from aquaculture and is transported to processing plants through ducks. The processing links include hair removal, segmentation, and classification. It is an important part of automatic product quality and safety information acquisition. According to the product category, they are stored in different cold stores; finally, they reach consumers through logistics and sales.

Domestic large-scale processing of duck products mostly uses semi-automated production technology, which is a combination of automated production lines and manual operations. In this way, the acquisition of product processing information mainly adopts the manual input method, the information collection efficiency is low, and it is easily affected by human factors, which has become a bottleneck problem in the improvement of product quality and safety management capabilities. In order to improve the quality and safety supervision of duck products, it is necessary to develop intelligent industrial information collection equipment. Through the automatic acquisition and reliable transmission of product quality information, the ability to collect industrial information can be improved and seamless acquisition and convergence of industrial information can be achieved.

Enterprises' product processing flow mainly consists of dozens of different processing steps, and each product has its own unique processing steps. To achieve accurate traceability of the product processing process, the information acquisition system needs to acquire information of livestock and poultry sources and specific processing information of products. This information includes more than ten different processing information, such as poultry farmers' information, employee information, weight information, initial processing information, deep processing links, refrigeration information, and packaging information. To obtain the above product information, the system needs to select the specific information collection method according to the actual processing situation, thus guaranteeing the real-time, accurate, and seamless collection of the whole processing flow information.

\section{System design}

\subsection{Enterprise processing model}

As shown in Fig. 1, in the processing enterprises, the processing steps of the same batch of products are basically the same, and the processing content and sequence of each link are basically the same. Combining the company's specific processes, the actual production process was simplified and abstracted, and a nondeterministic finite automaton (NFA) model was researched and constructed. The model structure is shown in Fig. 2. The variable information in the model is shown in Table 1.

As can be seen from Fig. 2, the circles represent different working conditions, the arrows pointing mean the trend of the state, and the information is the trigger for changing conditions. The NFA model can be described as $E=\{Q, X, M, \mathrm{q} 0, F\}$. Here, $Q$ is a finite set of states within the enterprise process model; $X$ is a set of triggering messages for different state transitions, that is, the trigger condition information of the state transition; $M$ represents the state transfer function of the state changes in the enterprise processing model, $M: X \times Q \rightarrow$ $Q$, that is, the current processing state receives a new trigger message to advance the processing step to the next step. The q0 represents the initial state of the enterprise process model, $\mathrm{q} 0 \in Q$. It is the state of the enterprise process has not yet started. And $F$ represents the end state of the enterprise processing model, it is included in $Q$. And it is the state that a batch of products are finished.

\subsection{The framework of the information collection system} At present, the structure of the Internet of Things is mainly divided into the following categories [17, 18]:

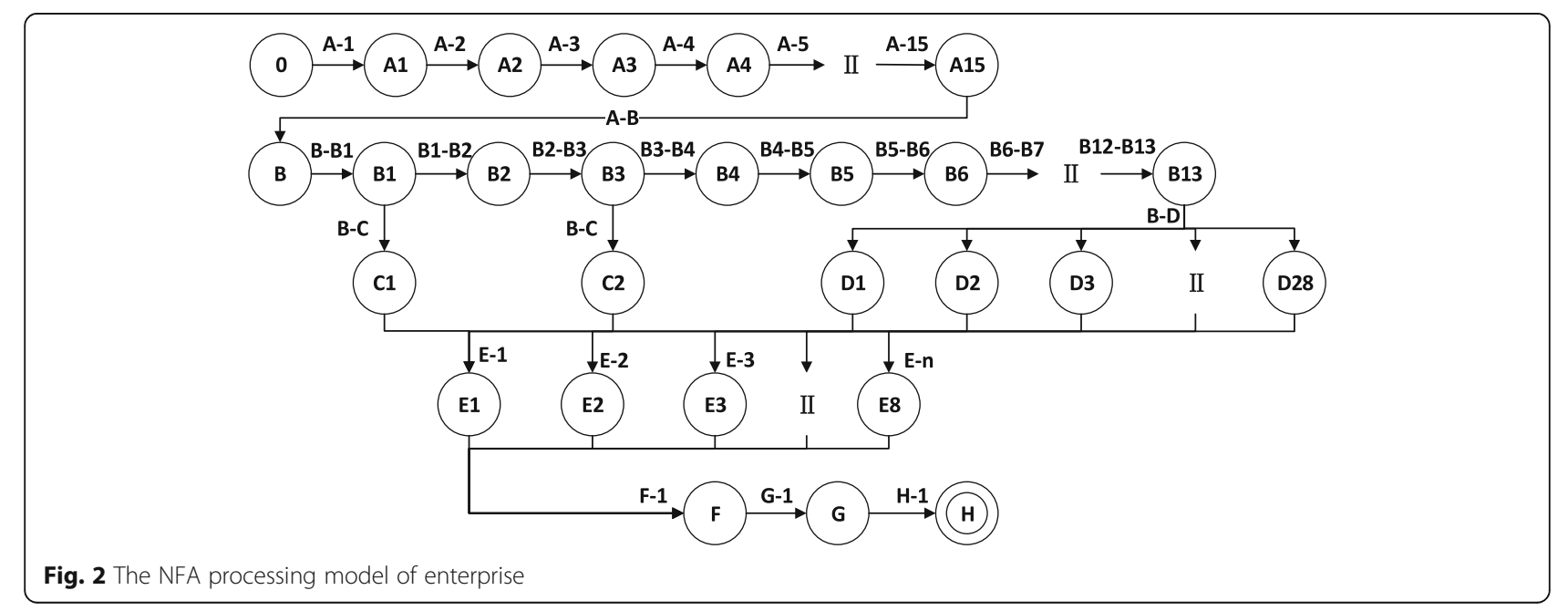


Table 1 The information in the NFA processing model

\begin{tabular}{ll}
\hline Variable & Name \\
\hline O & Start \\
A & First processing links-1 \\
B & First processing links-2 \\
C & By-product processing links \\
D & Main product processing links \\
E & Refrigeration links \\
F & Packaging links \\
G & Storage link \\
H & End \\
$X-Y$ & Trigger message \\
\hline
\end{tabular}

The USN-based five-tier structure model includes sensor networks, access networks, basic network facilities, middleware, and application platforms. The physicalnet's four-layer structure includes service provider network, gateway layer, coordination layer, and application layer. And the concept model of GB/T 33474-2016 structure implemented in 2017 mainly consists of user domain, target object domain, perception control domain, service provision domain, operation and maintenance management domain, and resource exchange domain.

The duck product traceability information acquisition system based on the Internet of Things aims to achieve real-time, accurate, and seamless information acquisition of the entire process. On the basis of a comprehensive analysis of the internal characteristics of the company, combined with the analysis results in 2.1 and the characteristics of different architecture systems of the Internet of Things, the GB/T 33474-2016 Internet of Things reference system was finally selected as the system architecture model.

This model can not only meet the functional requirements of the system, but also adopt the domain structure model, which is conducive to cross-domain and cross-industry resource sharing. The model consists of three parts: the perception control domain, the service domain, and the user domain. Perceptual control domain is the core of obtaining product processing information and controlling the process of product processing. It is mainly composed of several kinds of lower computer terminals. The service domain is the core of system storage, intelligent analysis, and early warning functions. The user domain is an important way for consumers to understand product processing information. At the same time, it is also an important window for enterprises to view real-time monitoring and manage basic data. The framework model of the system is shown in Fig. 3.

As shown in Fig. 3, the lower computer terminal relates to the host computer management software through the bus to realize data communication. The enterprise user system is connected to the host computer management software through the internal LAN to realize data sharing and data management. The consumer user system is connected to the host computer management software through Ethernet to achieve traceability data sharing. Due to the harsh processing

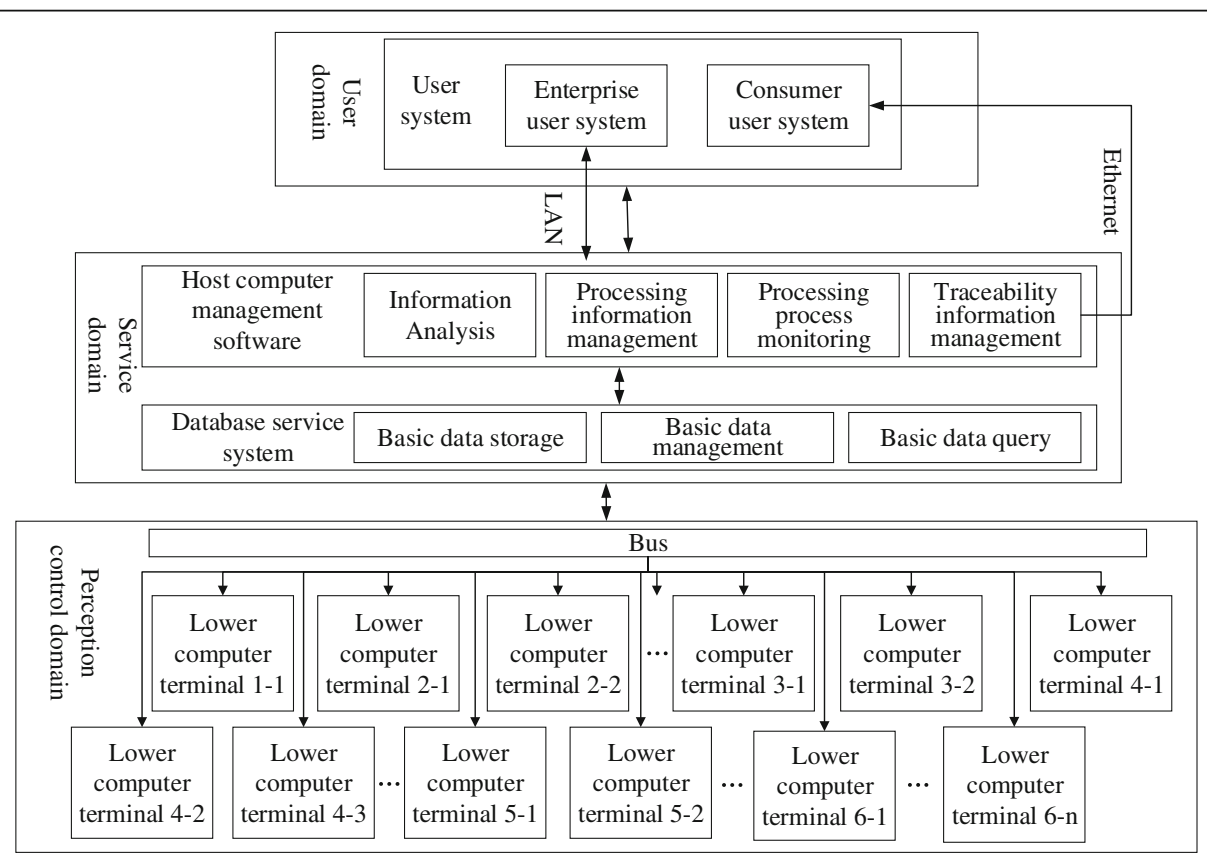

Fig. 3 Framework model of the information collection system 
environment and complex processing procedures, the Internet of Things is utilized to realize the accurate perception and seamless acquisition of different processing information based on the existing automation equipment in the enterprise.

\subsubsection{Perception control domain}

The perceptual control domain is an important part of the information collection system, and it is the fundamental guarantee for achieving product processing information acquisition. This domain is mainly composed of the lower computer terminals with different functions. As shown in Fig. 3, the lower computer terminals are distributed in different links and regions of the enterprise processing line. The concrete structure of the lower computer terminal is shown in Fig. 4.

As can be seen from Fig. 4, the lower computer terminal adopts the modular structure; the touch-screen module of the system realizes human-machine friendly interaction through voice and text. The integrated information acquisition module achieves real-time acquisition of different processing information in different processing areas by linking different information acquisition devices. The intelligent control module realizes the preprocessing of the collected information by using the core processing chip. The UHF RFID read and write module achieves accurate acquisition of machining information. The real-time communication module realizes the real-time communication between the perceptual control domain and the service domain. According to Fig. 3, the lower computer terminal is divided into six categories according to the different function and operating method, such as batch generation equipment, processing information acquisition equipment, weighing equipment, product information acquisition equipment, warehousing equipment, and packaging equipment. Different types of lower computer terminals correspond to different working states in Fig. 2, respectively.

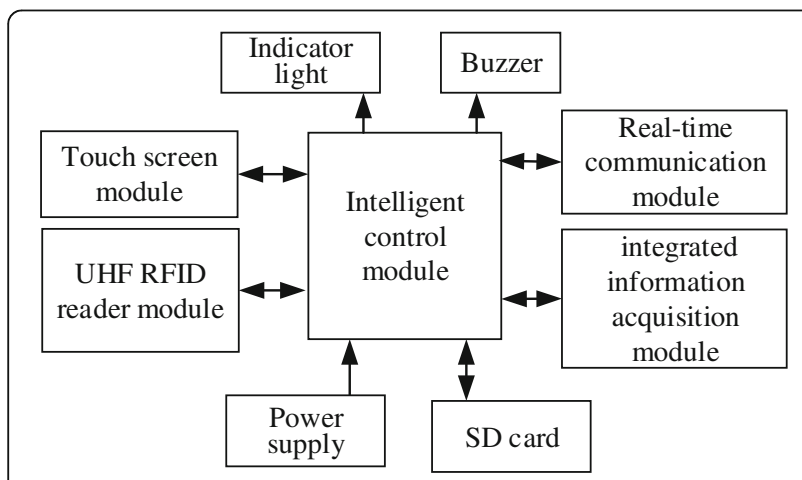

Fig. 4 The structure of the lower computer terminal

\subsubsection{Service domain}

Service domain is the core of analyzing and processing data of information collection system. This domain adopts $\mathrm{C} / \mathrm{S}$ service mode. By installing the corresponding system program, enterprise users can control the lower computer terminal in the sensing control domain. The system uses a unified server to provide production or technical guidance for enterprises. This domain is mainly composed of the host computer management system and the database server system. Its structure is shown in Fig. 5. The host management system is the corresponding client system of the system. It is mainly responsible for receiving and processing the product processing information uploaded by the lower computer and uploading the received information to the server. The server generates data that can be applied to the user system according to the original information to provide business services for the enterprise. The database service system provides basic services for the host computer management system by managing basic data.

From Fig. 5, the host computer management software in accordance with the different function can be divided into information analysis module, processing information management module, process monitoring module, traceability information management module, and information transceiver module. The host computer management software realizes the analysis and processing of all kinds of raw data, the real-time monitoring of product processing process, and the management of traceability information by controlling the work of each module. By integrating the information of all the lower computer terminals in the industrial chain, the host computer management software has formed an information flow covering the whole industrial chain.

Oracle data was used by the database management system. The system uses Oracle's huge data space and

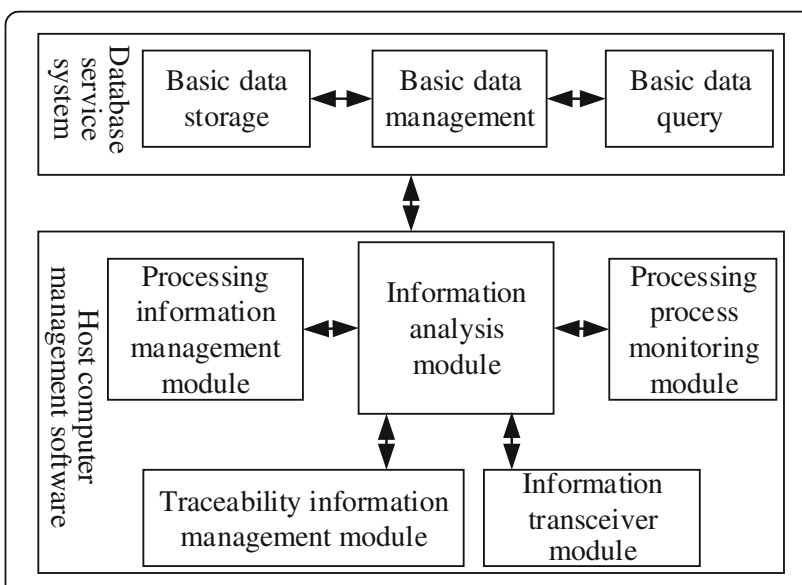

Fig. 5 The structure of the service domain 
better data management capabilities to provide basic services for PC management software.

\subsubsection{User domain}

User domain is the main way to achieve data sharing. Users can understand the product information by landing on the website. This domain uses the $\mathrm{B} / \mathrm{S}$ mode. Like the service domain, it uses the same server to provide information services. The system is divided into enterprise system and consumer system according to the different users' rights. The enterprise's user can view the processing information of all factory products through the enterprise system, and it can modify the basic data such as employee's information through the system. The consumers can view the basic information and quality inspection report information of the purchased products through the consumer system.

\section{System implementation}

\subsection{The workflow of the system}

The information collection system combines with the specific processing flow in the processing plant, and it realizes the information acquisition and real-time transmission of the whole processing industrial chain of duck products. It provides basic data support for traceability of processing information of duck products. Its specific processing flow is shown in Fig. 6.

As shown in Fig. 6, in order to realize the seamless collection and real-time transmission of product processing information, the lower machine terminal system adopts the autonomous information acquisition method. This function is mainly achieved through the UHF RFID read-write module to automatically obtain tag information. After obtaining the product processing information, the lower computer terminal transmits the collected information and the basic information to the host computer management software through the real-time communication module. After the host computer management software receives the information sent by the lower computer terminal, it first judges. If the information type is time information, it will feed back the current time information to the lower computer terminal. If it is product processing information, it will perform the following operations:

1. Check whether the information received from the lower terminal system is complete.

2. Analyze if the basic information is correct or not, such as employee number and terminal address information.

3. If the receiving information is complete and the basic information is correct, the software will analyze the type of receiving information and information content. If the receiving information exists in any one of the above errors, the software will analyze the type of error and error content.

4. Generate feedback information according to the analysis result in step 3 . If there is no error in the information, the feedback information will include the feedback command and the data information needed by the lower computer terminal. If there is an error in the information, the feedback information will contain the type of information error and the operation prompt.

5. The generated feedback information is transmitted to the lower computer terminal through the bus.

6. After the lower computer terminal receives the feedback information of the host computer management software, it first checks whether the

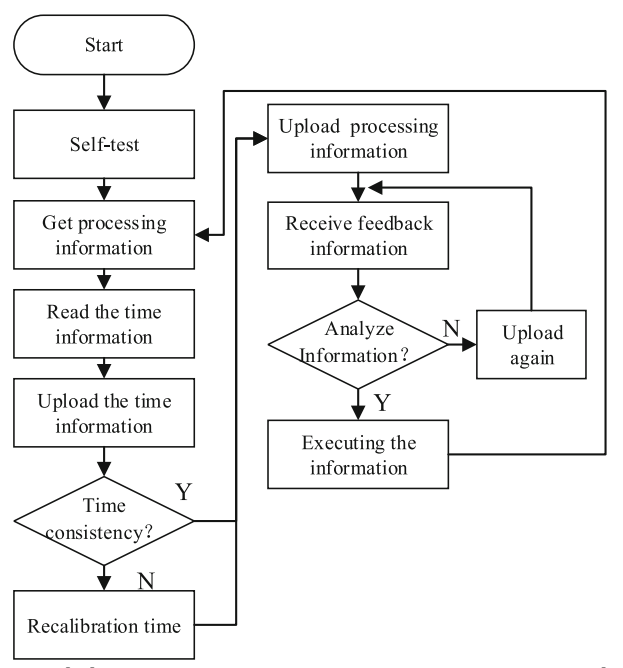

(a) The lower computer terminal

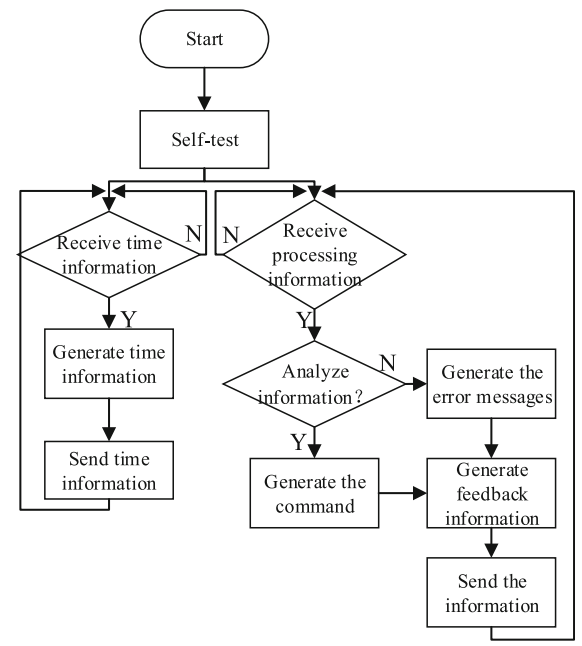

(b) The host computer management software

Fig. 6 The workflow of the system. a The lower computer terminal. b The host computer management software 
information content is complete and correct. Then, the content of feedback information is analyzed.

Finally, the related operations are performed according to the feedback content.

\subsection{The structure of the lower computer terminal}

As can be seen from Fig. 5, the intelligent control module is the key to achieve the accurate processing information of the lower computer terminal. To meet the functional requirements of the enterprise, the intelligent control module of the lower computer terminal adopts the self-developed central control panel. The structure is shown in Fig. 7. It includes many kinds of circuits, such as core control chip, storage circuit, clock circuit, signal conversion circuit, reset circuit, voltage stabilizing circuit, and external port circuit.

To improve system efficiency and data processing speed, the improved central control board circuit is adopted. The improved central control board can access the memory card of $8 \mathrm{G}$. By storing the algorithm and part of the data in the memory card, the system reduces the memory consumption of the control chip and avoids the loss of the set data caused by the sudden power failure. In addition, it also has an improved clock module circuit using the DS3231 chip. To ensure the stability of the clock module, this module uses independent power supply system to supply power. And the lower computer terminal communicates with the host computer management system by bus. To avoid the influence of the external circuit on data transmission, the system directly embedded the signal conversion circuit on the central control board. And Fig. 8 is the signal conversion circuit of the central control panel.

It can be seen from Fig. 5 that the lower computer realizes the real-time acquisition of relevant information through the integrated information acquisition module and the UHF RFID read-write module. The integrated

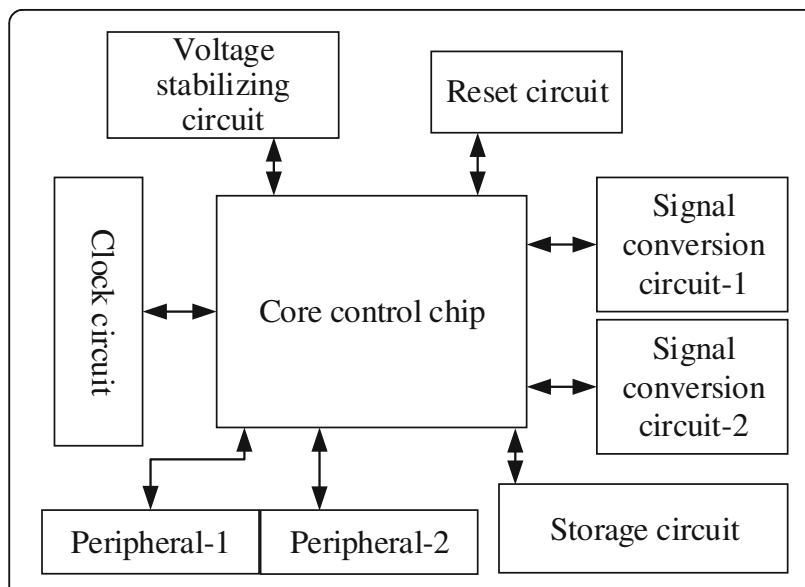

Fig. 7 The structure of the central control board information acquisition module can connect different types of sensing devices according to needs and can conveniently collect various types of information in different links. For example, in the deep processing stage, the product was subdivided into more than 30 different processes, and some products were packaged in the process. Each packaging bag is pre-printed with a twodimensional code, and the integrated information collection module scans the two-dimensional code to achieve mapping with product information to achieve accurate traceability of duck products. The UHF RFID reader module is a functional unit for the lower machine to obtain duck product information through RF signals. The functional unit is connected to the central control board of the lower computer through a serial communication interface. The RFID module has stable operation, reliable performance, long read distance characteristics, and the like.

\subsection{Information processing}

Because of the harsh processing environment of products and the variety of product processing information, it is easy to form problems such as channel blockage, interference signals, and information loss. To solve the above problems, several solutions have been proposed by the system. Here are some examples.

\subsubsection{Information processing of the losing information}

Take the automatic weighing of duck products as an example to analyze the information collection process and information processing methods. The quantity and weight information of the product for the lower computer terminal are obtained from the weight sensor and the quantity sensor. As the weight sensor and the quantity sensor continuously send data information to the integrated information sensing module, data information of other key serial ports cannot be obtained. This problem is a complex system problem. The complex event processing (CEP) method can treat the system data as different types of events. By analyzing the relationships between events, the event relationship sequence database can be established to solve the problem quickly. Therefore, CEP method is applied to solve this problem.

The CEP approach mainly consists of three parts. First, define the data information and its type during this operation. Then, define the basic events in this process. Finally, transform the atomic event into a complex semantic event and deal with it.

Before analyzing the event, the system needs to analyze the data type that the lower computer system obtains. The effective identification information required for weighing links includes three parts: batch start information, total weight and quantity information of batches, and batch ending information. Combined with 


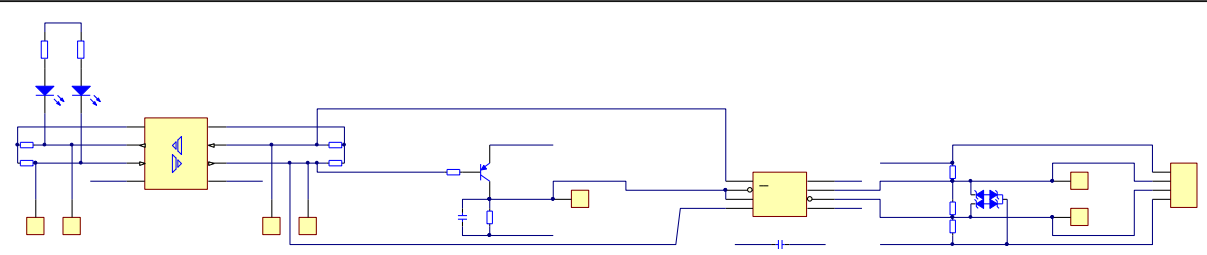

Fig. 8 The structure of the signal conversion circuit

the analysis results of the actual working process of the system, four kinds of event information are defined. They are batch start event (START), grouping information collection event (GROUPING_INFORMATION), final message collection event (FINAL_INFORMATION), and batch end event (END). The START and END represent events that the lower machine terminal system gets batch start/end identification information. The GROUPING_INFORMATION represents the event of the packet information obtained from the automatic electronic scale by the comprehensive information collection module in the lower computer terminal. The FINAL_INFORMATION represents the event of the total weight and quantity information obtained from the automatic electronic scale by the comprehensive information collection module in the lower computer terminal.

Analyze the specific processing process of the weighing link and build the NFA model for it as shown in Fig. 9. Map the events in this process to the state in NFA. As shown in Fig. 9, the NFA contains five states: " 0 ," " 1, , " 2, , " 3 ," and " 4 ." " 0 " is the system ready state. " 1 " is the batch start state. " 2 " is the packet information collection state. " 3 " is the final information collection state. And "4" is the batch end state.

If the system scans the START event when it is in "0" state, it will enter the " 1 " state. When it enters the " 1 " state, if the GROUPING_INFORMATION event is scanned, the system will enter the "2" state. When it scans the FINAL_INFORMATION event in the "2" state, it will enter the " 3 " state. After the system enters the " 3 " state, it will enter the "4" status when the END event is scanned. Entering the "4" status means that a batch of information has been completed. The system will transition to the "1" state when the system scans a START event in the state "4." This means the beginning of a new batch of information. As can be seen from Fig. 9, there is a self-loop identified named "GROUPING_INFORMATION" above the state "2," which means that if the GROUPING_INFORMATION event is scanned under state "2," there will be no transition occurring. That means the state returns to the original state constant.

\subsubsection{Information processing of signal interference}

The lower computer terminal obtains the processing information of the product through the UHF RFID reading and writing module. However, RFID readers do not have a tag filter function, which means that RFID readers repeatedly read the same tag information. This poses great difficulties for the accurate acquisition of product processing information. To solve this problem, an RFID tag filtering method was proposed. The specific process is as follows.

The system selects the inventory mode of UHF RFID reader. In this mode, the device will filter the duplicate label information while automatically obtaining the identification information. This means that in the inventory mode, the system will define an interval $\mathrm{T}(T=1 \mathrm{~min})$, and the reader sends a command to the read label to invert its mark. Thus, in the next $T$ time, the stored label will no longer respond to the inventory command, so that the reader can get the information of the label that has not been stored. At the same time, the intelligent control module will save the acquired new RFID label information into the memory card. When the intelligent control module receives the RFID label information uploaded by the UHF RFID reader module, the intelligent control module will use the $\mathrm{B} / \mathrm{B}+$ tree search

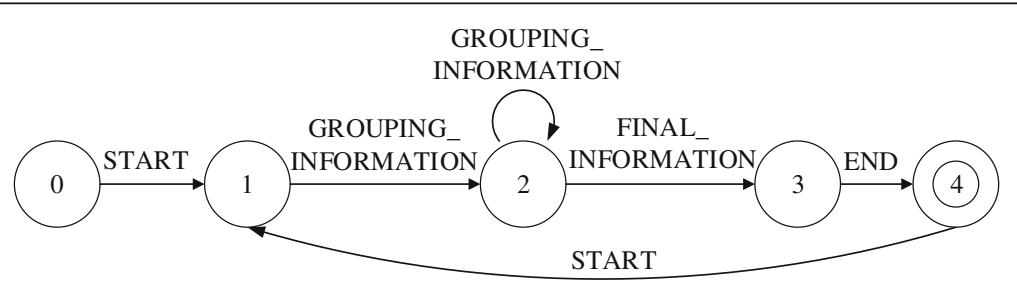

Fig. 9 The NFA of weighed link 
algorithm to find out whether the label information is in the memory card file. If this information has already existed, the system will automatically filter out the label information. Instead, the system will store the label information into the memory card. Then, the system will transmit the label information and the basic information to the host computer management software by the real-time communication module. The data in the memory card will be automatically emptied at 8 AM every day to ensure that the label information obtained by the lower computer terminal within a day will not be repeated.

\section{The deployment and application of the system}

The system has been successfully run in the enterprise. To obtain the information from the entire process of product processing, dozens of lower machine terminals have been deployed in the processing plant. They are distributed in all links of product processing, such as slaughter to deep processing, refrigeration, packaging, and warehousing. After half a year's test, operation, and debugging, the system has a stable and reliable overall performance, and the information collection is fast and accurate. It provides strong data support for enterprises to monitor the quality of products and provides basic data for the traceability of duck products. The system display interface is shown in Fig. 10.

\section{Results and discussion}

In addition to most large-scale duck processing companies, there are also small, privately run duck processing plants. In order to further promote the system in the entire industry and meet the economic burden of small businesses, the system needs to reduce costs without affecting the functions. In addition, the human-computer interaction of the system needs to be further improved. In particular, the individual links that require manual operation by employees need to further simplify the operation steps and meet the needs of users.

\section{Conclusions}

The duck product information collection system based on the Internet of Things is an information collection system covering the whole process of product processing. Through terminal research and bus-based industrial process perception network construction, industrial information collection, reliable transmission, and intelligent processing are realized.

According to the specific contents and requirements of different processing links, a special information collection terminal suitable for different requirements and contents has been developed, which realizes the automatic collection of information in every link and high-efficiency input. The application of terminal not only improves the speed of information input, but also helps to achieve seamless connection of information.

In addition to product quality and safety information entry, the system can also automatically enter other information such as operators and managers, which will help the company's fine management and product quality and safety incidents.

The system implementation has significantly improved the quality and safety management capabilities of the company's products. To achieve the

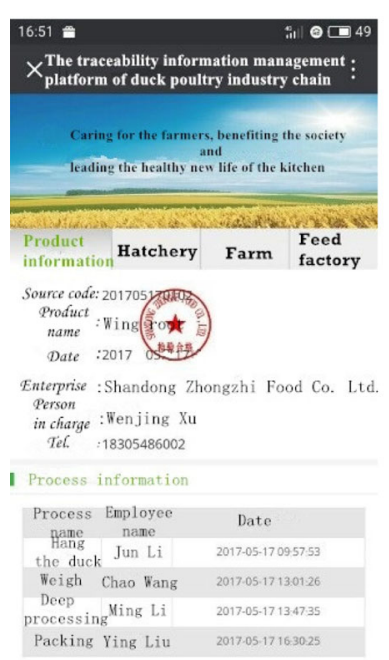

(a) The consumer user system
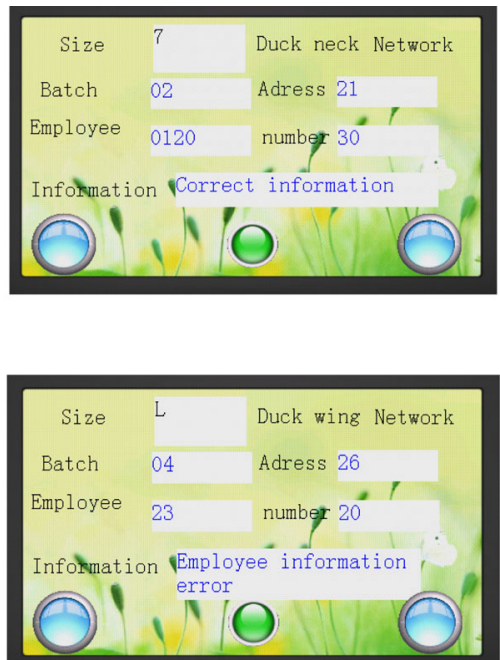

(b) The lower computer terminal

Fig. 10 The system display interface. a The consumer user system. b The lower computer terminal 
popularization and promotion of the system, it is necessary to further improve the terminal's perception ability and information preprocessing capability and improve the man-machine interaction friendliness of the terminal; the bus network structure still needs to be further improved. The flexibility and extensibility of the system improve the system's adaptability, further optimize the system structure, and improve the system's cost performance.

\section{Abbreviation}

CEP: Complex event processing; NFA: Nondeterministic finite automaton; RFID: Radio frequency identification

\section{Acknowledgements}

This work was supported by the research project "Major Data Application Technology Research and Integration Based on Livestock and Poultry Industry" of Shandong Province Major Agricultural Technological Innovation Project in 2017 and Shandong Independent Innovation and Achievements Transformation project (2014ZZCX07106)

\section{Funding}

The authors acknowledge the Shandong Independent Innovation and Achievements Transformation project (2014ZZCX07106).

\section{Authors' contributions}

LL is the main writer of this paper. She proposed the main idea, deduced the information collection system of duck products based on loT, and completed the design and implementation of the system. PL and FW built this big data platform framework. RZ and MY helped to modify the content of the article. XY helped to finish the experiment. All authors read and approved the final manuscript.

\section{Competing interests}

The authors declare that they have no competing interests.

\section{Publisher's Note}

Springer Nature remains neutral with regard to jurisdictional claims in published maps and institutional affiliations.

\section{Author details}

'College of Information Science and Engineering, Shandong Agricultural University, Tai'an 271018, China. ${ }^{2}$ Shandong Agricultural University, Tai'an 271018, China.

Received: 7 March 2018 Accepted: 7 May 2018

Published online: 18 May 2018

\section{References}

1. Welbourne $E$, et al. Building the internet of things using RFID: the RFID ecosystem experience[J]. IEEE Internet Comput., 2009, 13(3):48-55.

2. Broll G, et al. Perci: pervasive service interaction with the internet of things[]]. IEEE Internet Comput., 2009, 13(6):74-81.

3. Kranz $\mathrm{M}$, et al. Embedded interaction: interacting with the internet of things[]]. IEEE Internet Comput., 2010,14(2):46-53.

4. Thakur M, Hurburgh C R. Framework for implementing traceability system in the bulk grain supply chain[J]. J. Food Eng., 2009, 95(4):617-626.

5. Alexandros Kaloxylosa, Robert Eigenmannc, Frederick Teye, et al. Farm management systems and the future Internet era[J]. Comput. Electron. Agric.,2012,89(11):130-144.

6. Sébastien Pouliot, Daniel A Sumner. Traceability, liability, and incentives for food safety and quality[J]. Am. J. Agric. Econ.2008,90(1): 15-27.

7. Pang Chao, He Dongjian, Li Changyue, et al. Method of traceability information acquisition and transmission for dairy cattle based on integrating of RFID and WSN[]]. Transactions of the CSAE, 2011, 27(9): 147-152.

8. Ma Congguo, Zhao De'an, Liu Yefei, et al. Overall monitoring of pork industrialization production and development of traceability system[J]. Transactions of the CSAE, 2008,24(9):121-125.
9. Ren Shougang, Xu Huanliang, Li An, et al. Meat-productions tracking and traceability system based on internet of things with RFID and GIS[J]. Transactions of the CSAE, 2010, 26(10): 229-235.

10. Mc Inerney B, Corkery G, Ayalew $G$, et al. Preliminary in vivo study on the potential application of a novel method of e-tracking to facilitate traceability in the poultry food chain[J].Computers \& Electronics in Agriculture, 2011, 77(1):1-6.

11. Stanford K, Stitt J, Kellar J A, et al. Traceability in cattle and small ruminants in Canada.[J]. Rev. Sci. Tech., 2001, 20(2):510.

12. Sugiura $\mathrm{K}$, Onodera T. Cattle traceability system in Japan for bovine spongiform encephalopathy.[J]. Vet. Ital., 2008, 44(3):519.

13. Jianying Feng, Zetian Fu, Zaiqiong Wang, Mark Xu, Xiaoshuan Zhang. Development and evaluation on a RFID-based traceability system for cattle/ beef quality safety in China[J]. Food Control, 2013, 31(2):314-325.

14. Bevilacqua M, Ciarapica F E, Giacchetta G. Business process reengineering of a supply chain and a traceability system: A case study[]]. Journal of Food Engineering. 2009;93(1):13-22.

15. Hongwu Bai, Guanghong Zhou, Yinong Hu, Aidong Sun, Xinglian Xu, Xianjin Liu, Changhua Lu. Traceability technologies for farm animals and their products in China[J]. Food Control. 2017;79:35-43.

16. Zhao J, Zhu $C, X u Z$, et al. Microsatellite markers for animal identification and meat traceability of six beef cattle breeds in the Chinese market[]]. Food Control, 2017, 78:469-475.

17. Choi J, Park J, Park H D, et al. DART: Fast and efficient distributed stream processing framework for internet of things[]]. ETRI J., 2017, 39(2):202-212.

18. Ambrosin M, Anzanpour A, Conti M, et al. On the feasibility of attribute-based encryption on internet of things devices[]]. IEEE Micro, 2016, 36(6):25-35.

\section{Submit your manuscript to a SpringerOpen ${ }^{\circ}$ journal and benefit from:}

- Convenient online submission

- Rigorous peer review

- Open access: articles freely available online

- High visibility within the field

- Retaining the copyright to your article

Submit your next manuscript at $\gg$ springeropen.com 